\title{
WHEEL SLIP CONTROL IN ABS BRAKES USING GAIN SCHEDULED CONSTRAINED LQR
}

\author{
Idar Petersen*, Tor A. Johansen ${ }^{* * *}$, Jens Kalkkuhl ${ }^{* * *}$ and Jens Lüdemann*** \\ *SINTEF Electronics and Cybernetics, N-7465 Trondheim, Norway. e-mail: Idar.Petersen@ecy.sintef.no \\ ${ }_{* *}$ Department of Engineering Cybernetics, Norwegian University of Science and Technology, N-7491 Trondheim, Norway. \\ *** DaimlerChrysler, Research and Technology, Alt-Moabit 96a, D-10559 Berlin, Germany.
}

Keywords: Automotive control, gain scheduling, nonlinear systems, robustness, stability

\begin{abstract}
A wheel slip controller for ABS brakes is formulated using an explicit constrained LQR design. The controller gain matrices are designed and scheduled on the vehicle speed based on local linearizations. A Lyapunov function for the nonlinear control system is dervied using the Riccati equation solution in order to prove stability and robustness with respect to uncertainty in the road/tyre friction characteristic. Experimental results from a test vehicle with electromechanical brake actuators and brake-by-wire show that high performance and robustness are achieved.
\end{abstract}

\section{Introduction}

An anti-lock brake system (ABS) controls the slip of each wheel to prevent it from locking such that a high friction is achieved and steerability is maintained. ABS controllers are characterized by robust adaptive behaviour with respect to highly uncertain tyre characteristics and fast changing road surface properties [1].

The introduction of advanced functionality such as ESP (electronic stability program), drive-by-wire and more sophisticated actuators offers both new opportunities and requirements for higher performance in ABS brakes. The contribution of the present work is a study of a model-based design of ABS controllers, see also [2], [3]. We consider electromechanical actuators [4] rather than hydraulic actuators, which allow continuous adjustment of the clamping force. Here, we design the wheel slip controller based on a recently developed explicit LQR design method that takes into account input and state constraints [5], see also [6]. The wheel slip dynamics are highly nonlinear. Despite this, our control design relies on local linearization and gain-scheduling. In order to analyse the effects of this simplification, we develop a Lyapunov based stability and robustness analysis. Results from experiments using a test vehicle are also included. Other studies of model-based wheel slip control in ABS can be found in [7], [8], [9], [10].

\section{Modelling}

In this section, we review a mathematical model of the wheel slip dynamics, see also [1], [8] and [7]. The problem of wheel slip control is best explained by looking at a quarter car model as shown in Figure 1. The model consists of a single wheel attached to a mass $m$. As the wheel rotates, driven by inertia of the mass $m$ in the direction of the velocity $v$, a tyre reaction force $F_{x}$ is generated by the friction between the tyre surface and the road surface. The tyre reaction force will generate a torque that initiates a rolling motion of the wheel causing an angular velocity $\omega$. A brake torque applied to the wheel will act against the spinning of the wheel causing a negative angular

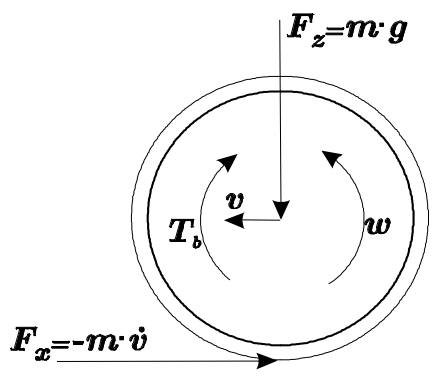

Figure 1: Quarter car forces and torques.

acceleration. The equations of motion of the quarter car are

$$
\begin{aligned}
m \dot{v} & =-F_{x} \\
J \dot{\omega} & =r F_{x}-T_{b} \operatorname{sign}(\omega)
\end{aligned}
$$

where

$v \quad$ horizontal speed at which the car travels

$\omega$ angular speed of the wheel

$F_{z} \quad$ vertical force

$F_{x}$ tyre friction force

$T_{b}$ brake torque

$r \quad$ wheel radius

$J \quad$ wheel inertia

The tyre friction force $F_{x}$ is given by

$$
F_{x}=F_{z} \cdot \mu\left(\lambda, \mu_{H}, \alpha\right)
$$

where the friction coefficient $\mu$ is a nonlinear function of

$$
\begin{array}{ll}
\lambda & \text { tyre slip } \\
\mu_{H} & \text { friction coefficient between tyre and road } \\
\alpha & \text { slip angle of the wheel }
\end{array}
$$

and the longitudial slip $\lambda$ defined by

$$
\lambda=\frac{v-\omega r}{v}
$$

$\lambda$ describes the normalised difference between horizontal speed $v$ and the speed of the wheel perimeter $\omega r$. The slip value of $\lambda=0$ characterises the free motion of the wheel where no friction force $F_{x}$ is exerted. If the slip attains the value $\lambda=1$, then the wheel is locked which means that it has come to a standstill.

The friction coefficient $\mu$ can span over a very wide range, but is differentiable with the property $\mu\left(0, \mu_{H}, \alpha\right)=0$ and $\mu\left(\lambda, \mu_{H}, \alpha\right)>0$ for $\lambda>0$. Its qualitative dependence on slip $\lambda$ is shown in Figure 2. The friction coefficient $\mu$ increases with slip $\lambda$ up to a value where it attains its maximum $\mu_{H}$. 

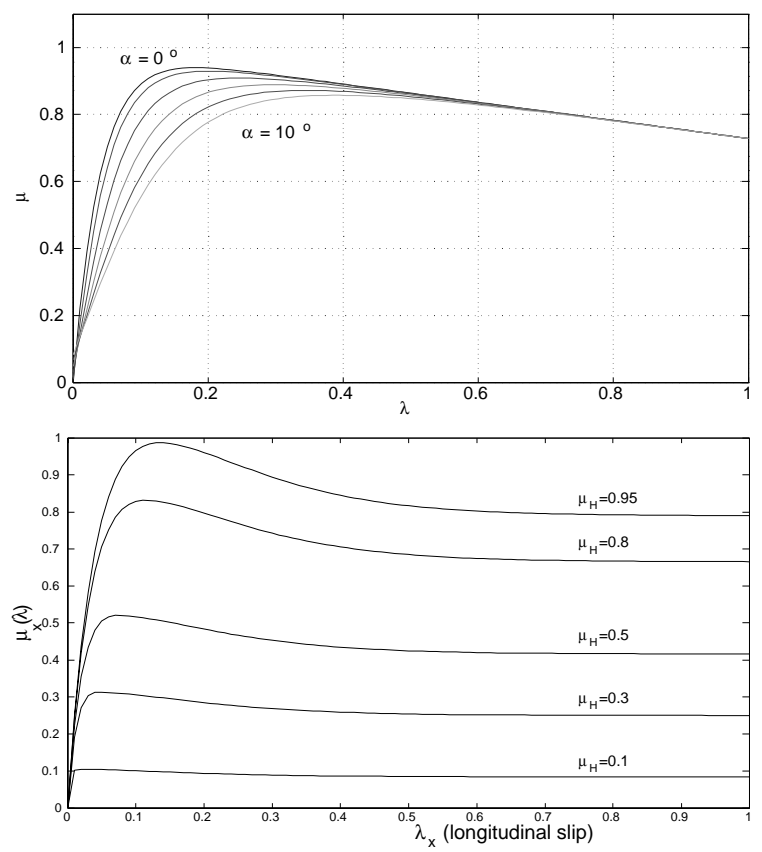

Figure 2: Tyre slip/friction curves $\mu\left(\lambda, \mu_{H}, \alpha\right)$

For higher slip values, the friction coefficient will decrease to a smaller value where the wheel is locked and only the sliding friction will act on the wheel. The dependence of friction on the road condition is shown in the bottom part of Figure 2. For wet or icy roads, the maximum friction $\mu_{H}$ is small and the right part of the curve is flatter. The tyre friction curve will also depend on the brand of the tyre. In particular, for winter tyres, the curve will cease to have a pronounced maximum.

If the motion of the wheel is extended to two dimensions, then the lateral slip of the tyre must also be considered. The slip angle $\alpha$ is where the wheel moves with velocity $v_{x}$ in the longitudinal direction and with a velocity $v_{y}$ in the lateral direction.

In this case, the longitudinal slip $\lambda_{x}=\frac{v_{x}-\omega r}{v}$ and the lateral slip $\lambda_{y}=\sin \alpha$ are distinguished as well as the corresponding friction coefficients $\mu_{x}$ and $\mu_{y}$. The top part of Figure 2 shows the dependence of the friction coefficient $\mu$ on the side slip angel $\alpha$. The side force $F_{y}$ depends greatly on the side slip angle $\alpha$. For large side slip angles, the force gets smaller. In the sequel, for simplification purposes unless otherwise stated, the side slip angle will be considered to be zero with $\mu_{x}=\mu$ and $v_{x}=v$.

Using (1)-(4) we get for $v>0$ and $\omega \geq 0$

$$
\begin{gathered}
\dot{\lambda}=-\frac{1}{v}\left(\frac{1}{m}(1-\lambda)+\frac{r^{2}}{J}\right) F_{z} \mu\left(\lambda, \mu_{H}, \alpha\right)+\frac{1}{v} \frac{r}{J} T_{b} \\
\dot{v}=-\frac{1}{m} F_{z} \mu\left(\lambda, \mu_{H}, \alpha\right)
\end{gathered}
$$

Note that when $v \rightarrow 0$, the dynamics of the open loop system (from $T_{b}$ to $\lambda$ ) becomes infinitely fast with infinite gain. This leads to a loss of controllability and the slip controller must be switched off for small $v$.

Proposition 1 Consider the system (5)-(6) with $T_{b}(t) \geq 0$ for all $t \geq 0$. If $v(0)>0$ and $\lambda(0) \in[0,1]$, then $\lambda(t) \in[0,1]$ and $\dot{v}(t) \leq 0$ for all $t \geq 0$ where $v(t)>0$.
Proof. Note that $\lambda(t)$ is a continuous trajectory. Hence, the possibile escape points are $\lambda=0$ and $\lambda=1$. Consider first $\lambda=0$. Since $\mu(0)=0$, it follows from (5) that $\dot{\lambda}=\frac{r}{v J} T_{b} \geq 0$ due to $T_{b}>0$. Hence, $\lambda(0) \geq 0$ implies $\lambda(t)>0$ for all $t>0$. Consider next $\lambda=1$. Then, $\bar{\omega}=0$ and from (2) it follows that $\dot{\omega} \geq 0$ due to the discontinuity $\operatorname{sign}(\omega)$ in (2). From (4), we conclude that $\dot{\lambda} \leq 0$ and $\lambda(0) \leq 1$, which implies $\lambda(t) \leq 1$ for all $t \geq 0$. Finally, note that $\dot{v} \leq 0$ from (1) because $F_{x} \geq 0$ for $\lambda \in[0,1]$.

\section{Control problem}

The control problem is essentially to control the value of the longitudinal slip $\lambda$ to a given setpoint $\lambda^{*}$ that is either constant or commanded from a higher-level control system such as ESP (electronic stability program). The controller must be robust with respect to uncertainties in the tyre characteristic and variations in the road surface conditions.

The control input is the clamping force $F_{b}$ that is related to the brake torque as $T_{b}=k_{b} F_{b}$. A maximum or a minimum force can be applied to the brake pads by the actuator during braking. The (small) minimum is to ensure that the pads are positioned close to the brake disk (no air-gap). Maximum is what the actuator is capable of. There is also a rate limit at how fast the torque can be changed by the actuator.

Integral action or adaptation must be incorporated to remove errors due to model inaccuracies, in particular the road/tyre friction coefficient $\mu_{H}$. It is essential that the controller maintains a high performance and is robust w.r.t. to any road/tyre friction curve that can be encountered cf. Figure 2.

\section{Gain-scheduled LQRC controller design and analysis}

The dynamics of the wheel and car body are given by (5) and (6), respectively. Due to large differences in inertia, the wheel dynamics and car dynamics will evolve on significantly different time scales. The speed $v$ will change much more slowly than the slip $\lambda$ and is therefore a natural candidate for gain scheduling. Thus, for the control design, we consider only (5) and regard $v$ as a slow time-varying parameter. A constrained LQR controller [5] is applied. This requires a nominal linearized model for design.

\subsection{Linearized dynamics}

Let $\left(\lambda^{*}, \hat{T}_{b}\right)$ be an equilibrium point for (5) defined by the constants $\alpha^{*}, F_{z}^{*}$ and $\mu_{H}^{*}$, where $\lambda^{*}$ is the desired slip

$$
\hat{T}_{b}=\left(\frac{J}{m r}\left(1-\lambda^{*}\right)+r\right) F_{z}^{*} \mu\left(\lambda^{*}, \mu_{H}^{*}, \alpha^{*}\right)
$$

The linearized slip dynamics are given by

$$
\dot{\lambda}=\frac{\alpha_{1}}{v}\left(\lambda-\lambda^{*}\right)+\frac{\beta_{1}}{v}\left(T_{b}-\hat{T}_{b}\right)+\text { h.o.t. }
$$

where $\alpha_{1}$ and $\beta_{1}$ are linearization constants given by

$$
\begin{aligned}
\alpha_{1} & =-F_{z}^{*}\left(\frac{1}{m}\left(1-\lambda^{*}\right)+\frac{r^{2}}{J}\right) \frac{\partial \mu}{\partial \lambda}\left(\lambda^{*}, \mu_{H}^{*}, \alpha^{*}\right) \\
& +F_{z}^{*} \frac{1}{m} \mu\left(\lambda^{*}, \mu_{H}^{*}, \alpha^{*}\right) \\
\beta_{1} & =\frac{r}{J}>0
\end{aligned}
$$


The wheel slip dynamics (5) can be written in the form

$$
\dot{x}_{2}=\frac{\phi\left(x_{2}\right)}{v}+\frac{\beta_{1}}{v}\left(T_{b}-T_{b}^{*}\right)
$$

where

$$
\begin{aligned}
\phi\left(x_{2}\right) & =-\left(\frac{1}{m}\left(1-\lambda^{*}-x_{2}\right)+\frac{r^{2}}{J}\right) F_{z} \mu\left(x_{2}+\lambda^{*}, \mu_{H}, \alpha\right) \\
& +\frac{r}{J} T_{b}^{*}
\end{aligned}
$$

and $x_{2}=\lambda-\lambda^{*}$ and

$$
T_{b}^{*}=\left(\frac{J}{m r}\left(1-\lambda^{*}\right)+r\right) F_{z} \mu\left(\lambda^{*}, \mu_{H}, \alpha\right)
$$

It can be seen that this system has an equilibrium point given by $x_{2}=0, T_{b}=T_{b}^{*}$ and $\phi(0)=0$, and the linearized slip model (7) with a perturbation term written as follows

$$
\dot{x}_{2}=\frac{\alpha_{1}}{v} x_{2}+\frac{\beta_{1}}{v}\left(T_{b}-T_{b}^{*}\right)+\frac{\epsilon_{\mu}\left(x_{2}\right)}{v}
$$

where $\epsilon_{\mu}\left(x_{2}\right)=\phi\left(x_{2}\right)-\alpha_{1} x_{2}$.

\subsection{Wheel slip control with integral action}

Let the system dynamics be augmented with a slip error integrator $\dot{x}_{1}=\lambda-\lambda^{*}=x_{2}$

$$
\left(\begin{array}{c}
\dot{x}_{1} \\
\dot{x}_{2}
\end{array}\right)=A(v)\left(\begin{array}{c}
x_{1} \\
x_{2}
\end{array}\right)+B(v)\left(u-T_{b}^{*}\right)+W(v) \epsilon_{\mu}\left(x_{2}\right)
$$

where

$$
A(v)=\left(\begin{array}{cc}
0 & 1 \\
0 & \frac{\alpha_{1}}{v}
\end{array}\right), B(v)=\left(\begin{array}{c}
0 \\
\frac{\beta_{1}}{v}
\end{array}\right), W(v)=\left(\begin{array}{c}
0 \\
\frac{1}{v}
\end{array}\right)
$$

Since $T_{b}^{*}$ is assumed unknown, we have defined $u=T_{b}$, and the equilibrium point to be $x^{*}=\left(x_{1}^{*}, 0\right)^{T}$ and $u^{*}=T_{b}^{*}$ where $x_{1}^{*}$ is still unspecified and we get

$$
\dot{x}=A(v)\left(x-x^{*}\right)+B(v)\left(u-u^{*}\right)+W(v) \epsilon_{\mu}\left(x_{2}\right)
$$

Next, define the quadratic cost function for the purpose of local LQ design based on the nominal part of (15):

$$
\begin{gathered}
J(x(t), u[t, \infty))=\int_{t}^{\infty}\left(\left(x(\tau)-x^{*}\right)^{T} Q(v)\left(x(\tau)-x^{*}\right)\right. \\
\left.+\left(u(\tau)-u^{*}\right)^{T} R\left(u(\tau)-u^{*}\right)\right) d \tau
\end{gathered}
$$

Assuming constant $v$, the optimal control law is given by

$$
\hat{u}=K(v) x
$$

where the gain matrix $K(v)=-R^{-1} B^{T}(v) P(v)$ and we choose to neglect the unknown $x^{*}$ and $u^{*}$ which will be accounted for due to the integral action. The symmetric matrix $P(v)>0$ is defined by the solution to the algebraic Riccati equation for the design:

$$
\begin{aligned}
& P(v) A(v)+A^{T}(v) P(v) \\
& -P(v) B(v) R^{-1} B^{T}(v) P(v)=-Q(v)
\end{aligned}
$$

namely

$$
\begin{aligned}
& P_{1,1}(v)=\frac{\left(\alpha_{1}^{2}+\beta_{1}^{2} R^{-1}\left(Q_{2,2}(v)+\frac{2\left(Q_{1,1}(v) R\right)^{1 / 2}}{\beta_{1}} v\right)\right)^{1 / 2}}{\left(Q_{1,1}(v) R\right)^{-1 / 2} \beta_{1}} \\
& P_{1,2}(v)=\frac{v}{\beta_{1}}\left(Q_{1,1}(v) R\right)^{1 / 2} \\
& P_{2,2}(v)=\frac{\alpha_{1}}{\beta_{1}^{2} R^{-1}} v \\
& +\frac{\left(\alpha_{1}^{2}+\beta_{1}^{2} R^{-1}\left(Q_{2,2}(v)+\frac{2\left(Q_{1,1}(v) R\right)^{1 / 2}}{\beta_{1}} v\right)\right)^{1 / 2}}{\beta_{1}^{2} R^{-1}} v
\end{aligned}
$$

and the controller gains are

$K_{1}(v)=-\left(Q_{1,1}(v) R^{-1}\right)^{1 / 2}$
$K_{2}(v)=-\frac{\alpha_{1}+\left(\alpha_{1}^{2}+\beta_{1}^{2} R^{-1}\left(Q_{2,2}(v)+\frac{2\left(Q_{1,1}(v) R\right)^{1 / 2}}{\beta_{1}} v\right)\right)^{1 / 2}}{\beta_{1}}$

We introduce the saturated control

$$
u= \begin{cases}T_{b}^{\max }, & \hat{u}>T_{b}^{\max } \\ T_{b}^{\min }, & \hat{u}<T_{b}^{\min } \\ \hat{u}, & \text { otherwise }\end{cases}
$$

for some $T_{b}^{\max }>T_{b}^{\min } \geq 0$. Furthermore, we define the error (note that both $u$ and $\hat{u}$ depend on $v$ and $x$ )

$$
\epsilon_{s}(x, v)=\beta_{1}(u-\hat{u})
$$

With the definition $\epsilon(x, v)=\epsilon_{s}(x, v)+\epsilon_{\mu}\left(x_{2}\right)$ and assuming $x_{1}^{*}=T_{b}^{*} / K_{1}(v)$, the closed loop dynamics can be written as

$$
\dot{\tilde{x}}=(A(v)+B(v) K(v)) \tilde{x}+W(v) \epsilon\left(\tilde{x}_{2}, v\right)
$$

with $\tilde{x}=x-x^{*}$.

Proposition 2 Consider the system (5) with controller (19). Assume $R>0$ and the smooth matrix-valued function $Q$ satisfies $Q_{1,2}(v)=Q_{2,1}(v)=0, Q_{1,1}(v)>0, Q_{2,2}(v)>0$, $\frac{d Q_{1,1}(v)}{d v} \geq 0, \frac{d Q_{2,2}(v)}{d v} \geq 0$ for all $v>0$. Moreover, suppose

$$
\begin{aligned}
Q_{1,1}(v) & >\frac{P_{2,1}(v) C}{v}(1-\delta) \\
Q_{2,2}(v) \tilde{x}_{2}^{2}> & \left(\frac{2 \epsilon_{\mu}\left(\tilde{x}_{2}\right) P_{2,2}(v) \tilde{x}_{2}}{v}+\frac{P_{2,1}(v) \epsilon_{\mu}^{2}\left(\tilde{x}_{2}\right)}{v C}\right)(1-\delta) \\
D(v) & =P_{1,1}^{\prime}(v) P_{2,2}^{\prime}(v)-P_{1,2}^{\prime}(v) P_{2,1}^{\prime}(v)>0
\end{aligned}
$$

are satisfied for all $v>0, \tilde{x}_{2} \in\left[-\lambda^{*}, 1-\lambda^{*}\right]$ and some $C>0$ and $\delta \in(0,1)$. If $T_{b}^{\max }>T_{b}^{*}>T_{b}^{\text {min }}$, the equilibrium $\tilde{x}=0$ is uniformly exponentially stable.

Proof. Consider a neighbourhood $X^{0}$ of the origin in $R^{2}$ such that $\epsilon_{s}(\tilde{x}, v)=0$ for all $\tilde{x} \in X^{0}$ and $v>0$. Let a Lyapunov function candidate be

$$
V(\tilde{x})=\tilde{x}^{T} P(v) \tilde{x}
$$


Its time-derivative along trajectories of (21)

$$
\dot{V}=\tilde{x}^{T}\left(\frac{\partial P(v)}{\partial v} \dot{v}\right) \tilde{x}+\dot{\tilde{x}}^{T} P \tilde{x}+\tilde{x}^{T} P \dot{\tilde{x}}
$$

is found by substituting for (15), (17) and (18) in (25):

$$
\begin{aligned}
\dot{V} & =\tilde{x}^{T} \frac{\partial P(v)}{\partial v} \dot{v} \tilde{x}-\tilde{x}^{T} Q(v) \tilde{x} \\
& +\epsilon_{\mu}\left(\tilde{x}_{2}\right)\left(W^{T}(v) P(v) \tilde{x}+\tilde{x}^{T} P(v) W(v)\right)
\end{aligned}
$$

From Proposition 1, it is clear that $\dot{v} \leq 0$. Thus, the negativity of $\tilde{x}^{T}\left(\frac{\partial P(v)}{\partial v} \dot{v}\right) \tilde{x}$ requires $P^{\prime}(v)=\frac{\partial P(v)}{\partial v}>0$ for all $v>0$. For $P^{\prime}(v)$ to be positive definite, it is sufficient that $P_{1,1}^{\prime}(v)>0$ and $D(v)=>0$ which follows due to (24). Note that since $Q_{1,1}(v), Q_{2,2}(v), \beta_{1}>0$, it follows immediately that $P_{1,1}^{\prime}(v)>0$. Then, (26) becomes:

$$
\begin{aligned}
\dot{V} & \leq \epsilon_{\mu}\left(\tilde{x}_{2}\right)\left(W^{T} P(v) \tilde{x}+\tilde{x}^{T} P(v) W(v)\right)-\tilde{x}^{T} Q(v) \tilde{x} \\
& =-Q_{1,1}(v) \tilde{x}_{1}^{2}-Q_{2,2}(v) \tilde{x}_{2}^{2} \\
& +\frac{2}{v} \epsilon_{\mu}\left(\tilde{x}_{2}\right)\left(P_{2,2}(v) \tilde{x}_{2}+P_{2,1}(v) \tilde{x}_{1}\right)
\end{aligned}
$$

To obtain all $\tilde{x}_{1}$-terms in (27) in a quadratic form, we apply Young's inequality $2 a b \leq a^{2} / C+C b^{2}$. Hence,

$$
\begin{aligned}
\dot{V} & \leq-Q_{1,1}(v) \tilde{x}_{1}^{2}+\frac{P_{2,1}(v)}{v} \tilde{x}_{1}^{2} C-Q_{2,2}(v) \tilde{x}_{2}^{2} \\
& +\frac{2}{v} \epsilon_{\mu}\left(\tilde{x}_{2}\right) P_{2,2}(v) \tilde{x}_{2}+\frac{P_{2,1}(v)}{v} \frac{\epsilon_{\mu}^{2}\left(\tilde{x}_{2}\right)}{C}
\end{aligned}
$$

Due to (22) and (23) it follows that

$$
\dot{V} \leq-\delta Q_{1,1}(v) \tilde{x}_{1}^{2}-\delta Q_{2,2}(v) \tilde{x}_{2}^{2}
$$

and we conclude that the equilibrium is uniformly exponentially stable using Corollary 4.2, [11].

Inequality (22) states that the error weight $Q_{1,1}(v)$ must be sufficiently large, leading to a suffieciently large controller gain. Note that $P_{2,1}(v)$ depends on $Q_{1,1}(v)$, but it is evident that $P_{2,1}(v)$ increases with $\sqrt{Q_{1,1}(v)}$ such that (22) will indeed hold for a sufficiently large $Q_{1,1}(v)$, except when $v \rightarrow 0$.

Inequality (23) states that the error weight $Q_{2,2}(v)$ must also be sufficiently large, leading to a sufficiently high gain to stabilize the system. Note that $P_{2,2}(v)$ increases with $\sqrt{Q_{2,2}(v)}$ such that this is also possible, except for $v \rightarrow 0$.

Note that $Q_{2,2}(v)\left|\tilde{x}_{2}\right|$ is essentially chosen in (23) to dominate the perturbation $\epsilon\left(\tilde{x}_{2}\right)$. Inequality (23) should be checked with respect to the perturbations $\epsilon_{\mu}$ that are generated by all possible friction curves $\mu(\cdot)$ to ensure robust stability.

Inequality (24) can be seen to be non-restrictive since it will always be satisfied for $\alpha_{1}$ close to zero [3]. This corresponds to generating the nominal model by linearizing near the peak of the friction curve. Experience shows that high performance is indeed achieved this way. Note that for $\alpha_{1}=0$, no information on the friction curves is actually utilized in the control design.

The constant $C>0$ should be chosen to minimize conservativeness. However, the choice $Q_{1,2}(v)=Q_{2,1}(v)=0$ and
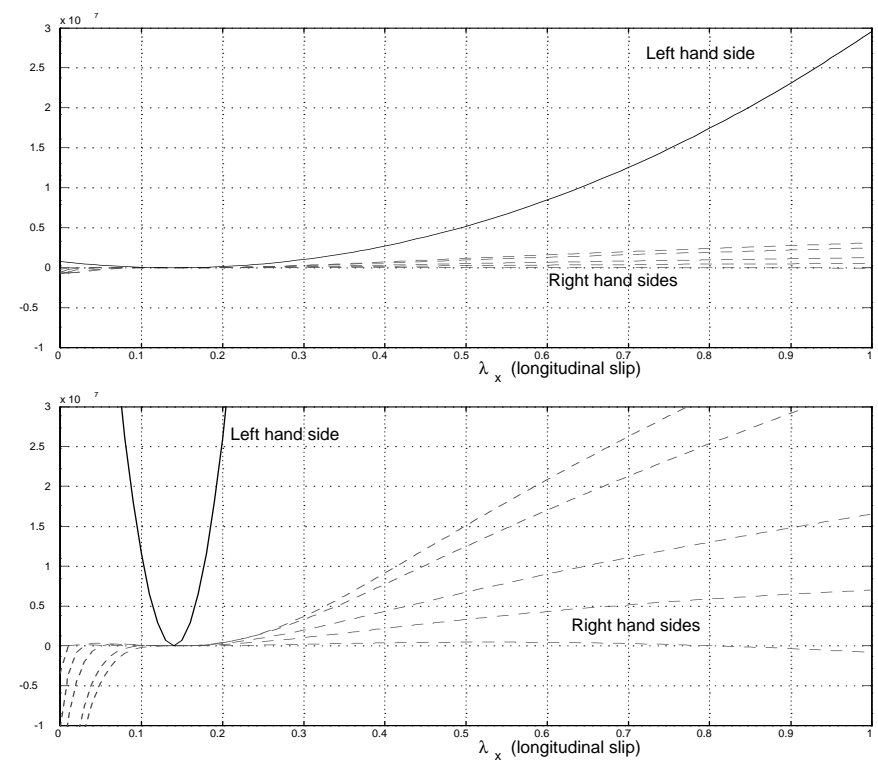

Figure 3: Illustration of the robust stability requirement, i.e. the left and right hand sides of eq. (23). The upper part is for $v=1 \mathrm{~m} / \mathrm{s}$ and the bottom part is for $v=32 \mathrm{~m} / \mathrm{s}$.

taking $P(v)$ from the solutions of the Riccati equation are possibly conservative.

The controller gain $K(v)$ depends on the speed (gain scheduling). From a practical point of view, a useful gain schedule is achieved by letting $\frac{d Q_{1,1}(v)}{d v}>0$ and $\frac{d Q_{2,2}(v)}{d v}>0$, as this reduces the gain as $v \rightarrow 0$. As mentioned earlier, this is necessary to avoid instability due to the unmodelled (actuator) dynamics since these tend to dominate as $v \rightarrow 0$.

An important aspect is the initialization of the integrator state $x_{1}$. Note that $x_{1}$ is a controller state that can be initialized arbitrarily, while $x_{1}^{*}$ is unknown since it depends on the road/tyre friction coefficient $\mu_{H}$. Hence, an intelligent initialization of $x_{1}(0)$ based on any a priori information on $\mu_{H}$ and possibly on the known $\tilde{x}_{2}(0)$ may significantly improve the transient performance [2].

\subsection{An idealized design example}

We consider a design example with the following parameters $m=450 \mathrm{~kg}, F_{z}=4414 \mathrm{~N}, r=0.32 \mathrm{~m}, J=1.0 \mathrm{~kg} \mathrm{~m} \mathrm{~m}^{2}$ and the friction model in the right part of Figure 2. Assuming $\lambda^{*}=0.14$ and the nominal design $\mu_{H}^{*}=0.8$ and $\alpha^{*}=0$, we get $\alpha_{1}=10.2$ and $\beta_{1}=0.32$. We choose $R=1$ and $Q(v)=\tilde{Q} v^{3 / 2}$ with $\tilde{Q}_{1,1}=6 \cdot 10^{9}$ and $\tilde{Q}_{2,2}=40 \cdot 10^{6}$. Note the scaling due to the different magnitudes of $T_{b}$ and $\lambda$. The choice for $Q(v)$ leads to a gain schedule with reduced gain as $v \rightarrow 0$, that is useful to avoid instability due to unmodelled acatuator dynamics as $v \rightarrow 0$.

Figure 3 shows that the robust stability requirement (23) is satisfied for all $\tilde{x}_{2} \in\left[-\lambda^{*}, 1-\lambda^{*}\right]$ for all the friction curves in the right part of Figure 2. Although curves are shown only for $v=1 \mathrm{~m} / \mathrm{s}$ and $v=32 \mathrm{~m} / \mathrm{s}$, we have verified that (24) is fulfilled for intermediate values of $v$. The control design is also satisfied the stability requirements (22) and (24), and we conclude robust uniform exponential stability of the equilibrium. 


\section{Implementation}

The experimental vehicle is a Mercedes E220 equipped with electromechanical disc brake actuators and a brake-by-wire system. A previous version of this system is described in [4]. The system consists of four independent servo controllers, one for each brake, and a central electronic control unit (ECU) where the wheel slip controllers run. With reference to the theoretical study presented in previous sections, we note the following implementation details:

- The actual control implementation is based on the discretization of the models and a discrete-time control design. The discrete-time model also takes into account communication delays on the brake-by-wire electronics. The sampling rate for the wheel slip control loop is $7 \mathrm{~ms}$.

- The electromechanical brake actuator is assumed to be linear,

$$
\tau \dot{F}_{b}=-F_{b}+\tilde{F}_{b}
$$

where $\tau$ is the time-constant and $\tilde{F}_{b}$ is the commanded clamping force. This is reasonable since it contains a local feedback loop. The model used for control design is augmented with a discretized version of (29) and the measured clamping force $F_{b}$ is used for feedback.

- The controller actually computes the change of the clamping force, rather than the clamping force itself. This is implemented in order to enjoy the benefits of velocity-based gain scheduling [12], as well as it easily account for the actuator constraint (see [5])

$$
-\dot{F}_{b}^{\max } \leq \dot{F}_{b} \leq \dot{F}_{b}^{\max }
$$

Anti-windup is implemented on the integrator state $x_{1}$.

- Gain scheduling is implemented by computing gain matrices for a finite number of operating points and then switching gain matrices. To achieve bumpless transfer the integrator is reset at the switching instants. The gain matrices are scheduled on both speed and slip. The scheduling on slip only has an effect for very small slip values, and will improve the transient performance when the wheel slip controller is activated.

- The slip $\lambda$ and speed $v$ are estimated online using an extended Kalman filter based on wheel speed $(\omega)$ and acceleration measurements.

- The ABS system monitors the commands given by the driver using the brake pedal. Essentially, we set $T_{b}^{\max }=$ $k_{b} F_{b}^{*}$ where $F_{b}^{*}$ is the clamping force commanded by the driver using the brake pedal. The slip controller is deactivated when the speed is below $1 \mathrm{~m} / \mathrm{s}$.

- The tuning of the implemented controller is similar to the tuning in the idealized set-up in section 4.3. More specifically, the dominant pole of the nominal linear closed loop is almost the same in both cases (about 12.0 for a typical $v)$.

\section{Experimental results}

The first test, Figure 4, is braking on dry asphalt, starting at $v(0)=21.5 \mathrm{~m} / \mathrm{s}$ and without any steering manoeuvers. The slip setpoint is $\lambda^{*}=0.09$ and we note that the regulation is highly accurate and satisfactory. When the speed approaches zero, significant variability in the slip emerges. Since the
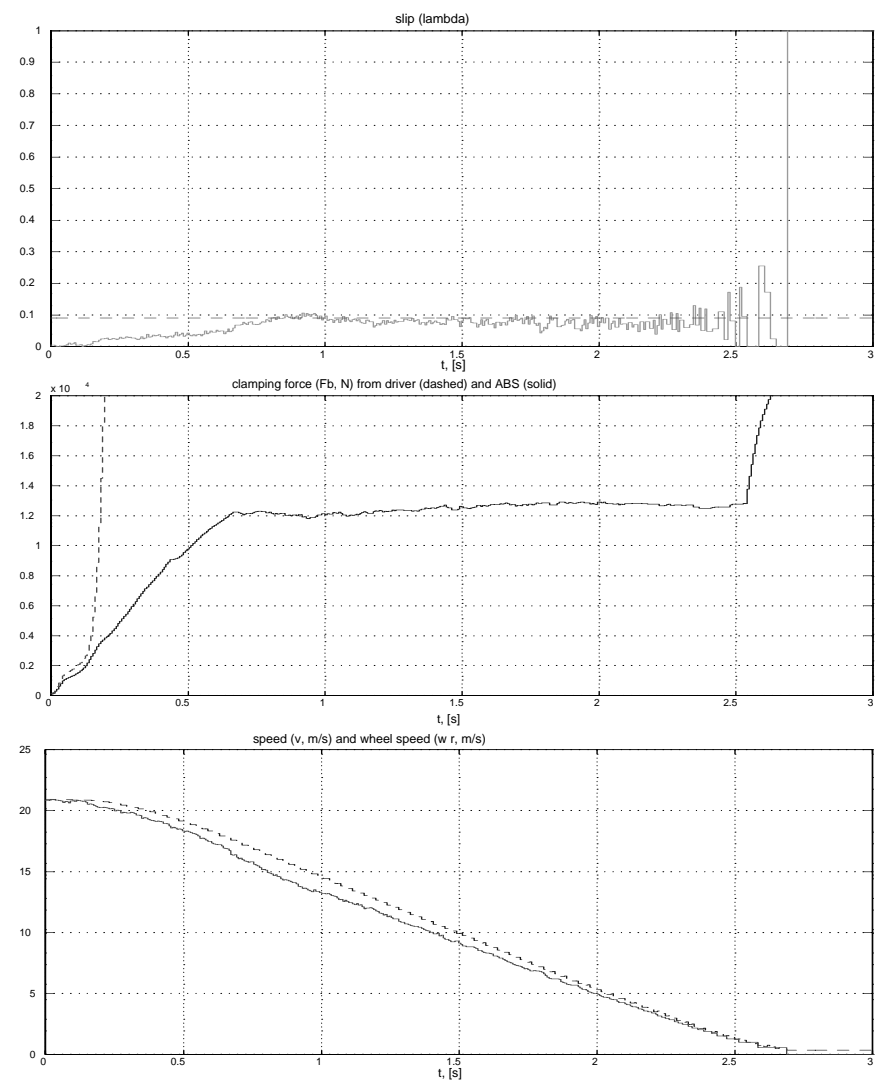

Figure 4: Experimental results with braking on dry asphalt.

clamping force does not oscillate, we conclude that this is actually sensor noise that is known to increase at the speed goes to zero. We also note that the initial transient is not satisfactory as the claming force does not increase fast enough so that the slip is too low and the resulting friction force is too low in the interval $t \in(0.2,0.7)$ leading to increased braking distance. This is due to the significant model inaccuracy in the low-slip region, and redesign of the slip controller is necessary for this region.

The second test, Figure 5, is braking on snow, starting at $v(0)=22.0 \mathrm{~m} / \mathrm{s}$ and without any steering manoeuvers. The slip setpoint is $\lambda^{*}=0.07$ and we note that the regulation is satisfactory. There are some small oscillations that we believe are due to unmodeled actuator phenomenon (non-linearities) that are expected to be more pronounced when operating at low clamping force levels due to low friction on snow.

The third test, Figure 6, is braking on a wet inhomogeneous surface (asphalt that is partially covered by a plastic coating and water) without any steering manoeuvers. The intial speed is $v(0)=22.0 \mathrm{~m} / \mathrm{s}$ and the slip setpoint is $\lambda^{*}=0.09$. We note that in this case there are significant transients in the slip and claming force. We still conclude that the regulation performance is highly satisfactory, since the surface is characterized by very large variations in the friction coefficient that cause large disturbances on the system.

\section{Discussion and Conclusions}

Using Lyapunov analysis and experimental verification we have shown good performance and robustness of a modelbased nonlinear wheel slip controller for ABS. In order to 

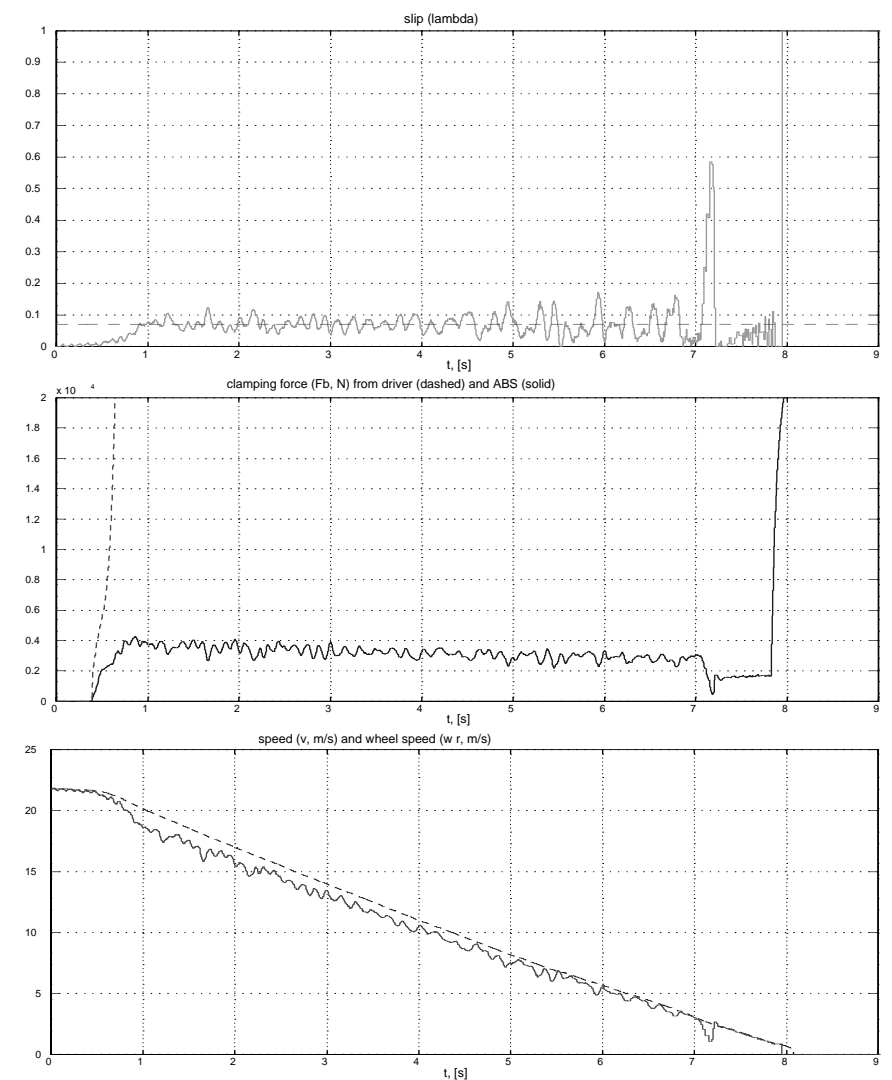

Figure 5: Experimental results with braking on snow.

achieve the robustness, the approach does not rely on knowledge of the tyre/road friction curve. Static uncertainty (due to unknown $\mu_{H}$ ) is eliminated using integral action, while dynamic uncertainty (due to unknown shape of $\mu(\cdot)$ ) is handled by a robust design with sufficient stability margin.

The results are somewhat prelimenary, and can be possibly be extended and improved. In particular, the analysis might take into consideration some of the details that are present in the implementation, but omitted from the current analysis. Also, the use of the Riccati-solution to define the Lyapunov function is not always the best choice. Finally, means for improving the transient performance of the controller (especially for low slip) are under investigation.

Although a detailed comparison with existing off-the-shelf ABS has not been conducted, the present results are encouraging, in particular when taking into account the modest time taken to design, tune and comission this model-based approach.

\section{Acknowledgements}

The work was sponsored by the European Commission under the ESPRIT LTR-project $28104 \mathrm{H}_{2} \mathrm{C}$.

\section{References}

[1] M. Burckhardt, Fahrwerktechnik: Radschlupf-Regelsysteme. Würzburg: Vogel Verlag, 1993.

[2] T. A. Johansen, J. Kalkkuhl, J. Lüdemann, and I. Petersen, "Hybrid control strategies in ABS," in American Control Conference, (Washington D.C.), 2001.

[3] I. Petersen, T. A. Johansen, J. Kalkkuhl, and
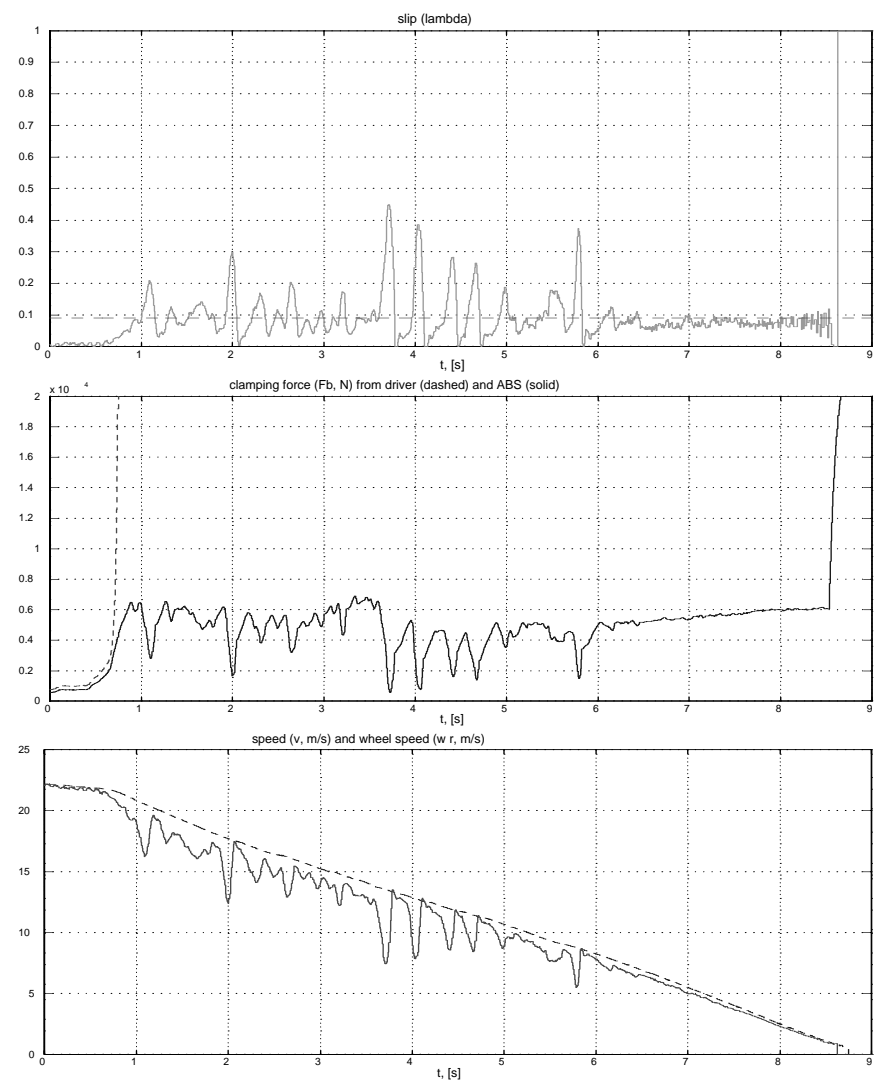

Figure 6: Experimental results with braking on a wet inhomogeneous suface.

J. Lüdemann, "Wheel slip control in ABS brakes using gain scheduled constrained LQR." http://www.itk.ntnu.no/ansatte/Johansen_Tor.Arne/ecc2001full.pdf, 2001.

[4] R. Schwarz, Rekonstruktion der Bremskraft Bei Fahrzeugen mit Elektromechanisch Betätigten Radbremsen. PhD thesis, Institut für Automatisierungstechnik der TU Darmstadt, 1999.

[5] T. Johansen, I. Petersen, and O. Slupphaug, "Explicit suboptimal linear quadratic regulation with state and input constraints," in IEEE Conference on Decision and Control, Sydney, 2000.

[6] A. Bemporad, M. Morari, V. Dua, and E. Pistikopoulos, "The explicit solution of model predictive control via multiparametric quadratic programming," in Proc. American Control Conference, Chicago, pp. 872-876, 2000.

[7] R. Freeman, "Robust slip control for a single wheel," Tech. Rep. CCEC 95-0403, University of California, Santa Barbara, 1995.

[8] S. Drakunov, Ü. Özgüner, P. Dix, and B. Ashrafi, "ABS control using optimum search via sliding modes," IEEE Trans. Control Systems Technology, vol. 3, pp. 79-85, March 1995.

[9] C. C. de Wit, R. Horowitz, and P.Tsiotras, "Model-based observers for Tire/Road contact friction prediction," in In New Directions in Nonlinear Observer Design (H. Nijmeijer and T. Fossen, eds.), pp. 23-42, Springer Verlag, May 1999.

[10] J. Yi, L. Alvarez, R. Horowitz, and C. C. de Wit, "Adaptive emergency braking control using a dynamical Tire/Road friction model," in IEEE Conference on Decision and Control, Sydney, 2000.

[11] H. K. Khalil, Nonlinear Systems. Prentice Hall, 1996.

[12] I. Kaminer, A. M. Rascoal, P. P. Khargonekar, and E. E. Coleman, "A velocity algorithm for the implementation of gainscheduled controllers," Automatica, vol. 31, pp. 1185-1191, 1995. 